\title{
Long Noncoding RNA DANCR Regulates Cell Proliferation by Stabilizing SOX2 mRNA in Nasopharyngeal Carcinoma
}

Qiang Li, ${ }^{*}$ Yiming Jiang, ${ }^{\dagger}$ Guansheng Zhong, ${ }^{\ddagger}$ Yanwei Lu, ${ }^{*}$ Tao Song, ${ }^{*}$ Yigan Zhang, ${ }^{\S}$ Jianhui Wu, ${ }^{\llbracket}$ Minjun Zhang, Xiaodong Liang, ${ }^{*}$ Liheng Zhou, ** Jianming Tang, ${ }^{*}$ and Haibo Zhang*

From the Department of Radiation Oncology,* Zhejiang Provincial People's Hospital, People's Hospital of Hangzhou Medical College, Hangzhou; the Department of Internal Medicine-Oncology, ${ }^{\dagger}$ The First Hospital of Jiaxing, Jiaxing; the Department of Breast Surgery, ${ }^{\ddagger}$ The First Affiliated Hospital, College of Medicine, Zhejiang University, Hangzhou; The First School of Clinical Medicine, ${ }^{\S}$ Lanzhou University, Lanzhou; the Department of The Otolaryngology, $"$ Zhongshan City People's Hospital, Zhongshan Affiliated Hospital of Sun Yan-Sen University, Zhongshan; the Graduate Department, Bengbu Medical College, Bengbu; and the Department of Breast Surgery, ${ }^{* *}$ Renji Hospital, School of Medicine, Shanghai Jiao Tong University, Shanghai, PR China

Accepted for publication

September 8, 2020.

Address correspondence to Haibo Zhang, M.D., or Jianming Tang, Ph.D., Department of Radiation Oncology, Zhejiang Provincial People's Hospital, People's Hospital of Hangzhou Medical College, Hangzhou, Zhejiang 310014, PR China. E-mail: zhbdoctor@163.com or 15900792812@163.com.
The long noncoding RNA DANCR (differentiation antagonizing non-protein coding RNA) displays aberrant expression in various cancers. However, its clinical value and functional mechanisms in nasopharyngeal carcinoma (NPC) remain poorly understood. We found that DANCR is dramatically up-regulated in human NPC, and that it is an indicator for poor survival prognosis. DANCR knockdown suppressed cell proliferation, colony formation in vitro, and tumorigenicity in vivo. Mechanistic analyses demonstrated that DANCR could bind to RNA-binding protein 3 (RBM3) protein and stabilize SOX2 mRNA, resulting in NPC cell proliferation. Our findings indicate that DANCR functions as an oncogene and a potential therapeutic target for NPC. (Am J Pathol 2020, 190: 2343-2354; https://doi.org/10.1016/j.ajpath.2020.09.005)
Nasopharyngeal carcinoma (NPC), a malignancy that occurs in the head and neck, is prevalent in southeast Asia and southern China. ${ }^{1,2}$ Although radiotherapy acts as the most effective treatment for NPC patients, ${ }^{3,4}$ recurrence and local advancement often lead to treatment failure. Therefore, finding a potential biomarker and understanding its predictive molecular mechanisms in NPC are urgent. New therapeutic targets are beneficial for clinical treatment strategies. Recent evidence reveals that the long noncoding RNAs (IncRNAs) regulate NPC tumorigenicity. ${ }^{5-7}$ Nevertheless, the precise mechanisms by which lncRNAs regulate NPC tumorigenicity are largely unknown.

The IncRNA DANCR (differentiation antagonizing nonprotein coding RNA), which is encoded on chromosome locus $4 \mathrm{q} 12$, is related to various cancers, including breast cancer, ${ }^{8}$ hepatocellular carcinoma, ${ }^{9}$ and NPC. ${ }^{10}$ DANCR was first reported to suppress epidermal cell differentiation. ${ }^{11}$ Other studies also indicated that DANCR sequesters miR-216a ${ }^{12}$ and miR-355 $\mathrm{a}^{13}$ in lung and cervical cancer, respectively. Moreover, Wen et $\mathrm{al}^{10}$ reported that DANCR stabilizes hypoxia-inducible factor- $1 \alpha$ mRNA by physically binding with the NF90/NF45 complex. DANCR is also demonstrated acting as a direct target of MYC. ${ }^{14}$ Interestingly, DANCR has recently been shown to activate phosphate 3-kinase catalytic subunit alpha (PIK3CA) transcription by increasing the serine $49 / 78$ phosphorylation of rketinoic acid receptor RXR-alpha (RXRA). ${ }^{15}$ Moreover, $D A N C R$ expression correlates with the prognosis of patients

\footnotetext{
Supported in part by Zhejiang Provincial People's Hospital Scientific Research Returned Foundation for the Excellent Youth grant ZRY2018B002 (J.T.), Zhejiang Provincial Nature Science Foundation of China grant LQ20H160063 (J.T.), Nonprofit Research Project of Jiaxing grant 2020AD30064 (Y.J.), Zhejiang Provincial Nature Science Foundation of China grant LQ20H160030 (G.Z.), and Zhejiang Provincial People's Hospital Scientific Research Returned Foundation for the Excellent Youth grant ZRY2018C009 (H.Z.).

Q.L. and Y.J. contributed equally to this work.

Disclosures: None declared.
} 
with NPC. ${ }^{10}$ Nevertheless, the function of DANCR in NPC tumorigenicity is largely unclear.

In the current study, we found that DANCR was dramatically up-regulated in NPC. Meanwhile, it could indicate poor survival prognosis for NPC patients. RNAsequencing (RNA-Seq) analysis suggested that DANCR regulated SOX2 expression. Furthermore, DANCR induced NPC cell proliferation, colony formation in vitro, and tumorigenicity in vivo through association with $R B M 3$ to stabilize SOX2 mRNA. Thus, our findings indicated that DANCR served as an oncogene in NPC and might provide a potential therapeutic target for NPC patients.

\section{Materials and Methods}

\section{Cell Lines}

The C666-1, SUNE-1, HNE-1, CNE1, and CNE2 (human NPC cell lines), as well as the NP69 (normal nasopharyngeal epithelium cell line), were purchased from the Cell Bank of Chinese Academy of Sciences Committee on Type Culture Collection (Shanghai, China). Through an STR DNA fingerprinting at Shanghai Biowing Applied Biotechnology Co., Ltd. (Shanghai, China), all cell lines were authenticated.

\section{Tissue Samples and Clinical Data Collection}

Ten pairs of freshly frozen NPC tumor and adjacent nontumor specimens were obtained from Zhongshan City People's Hospital. An additional 100 pairs of formalinfixed, paraffin-embedded NPC tumor and the adjacent normal specimens were also collected from Zhejiang Provincial People's Hospital. None of the specimens was collected from patients who had undergone chemotherapy or radiotherapy at the time of biopsy. The ethics consent was obtained from the Institutional Ethical Review Board of Zhejiang Provincial People's Hospital (institutional review board number 2020QT258) before commencing our analysis of these samples.

\section{RNA Preparation and Real-Time Quantitative PCR}

In brief, the RNA preparation and subsequent real-time quantitative PCR were conducted as previously described. ${ }^{16}$ Total RNA was extracted using TRIzol reagent (Invitrogen, Carlsbad, CA), and real-time quantitative PCR analyses were performed on the basis of the manufacturer's instructions (Thermo Fisher, Waltham, MA). All primer sequences are listed in Table 1.

\section{Construction of Recombinant Plasmids}

DANCR, RBM3, and SOX2 transcripts from NP69 cells were amplified by real-time PCR. After sequencing, these transcripts were subcloned into the pcDNA3.1 or pLVX-Puro
Table 1 Primers for qPCR Assays

\begin{tabular}{ll}
\hline Primer pairs & Sequence \\
\hline qPCR, GAPDH & F: 5'-GGAGCGAGATCCCTCCAAAAT-3' \\
& R: 5'-GGCTGTTGTCATACTTCTCATGG-3' \\
qPCR, SOX2 & F: 5'-GCCGAGTGGAAACTTTTGTCG-3' \\
& R: 5'-GGCAGCGTGTACTTATCCTTCT-3' \\
qPCR, RBM3 & F: 5'-GAGGGCTCAACTTTAACACCG-3' \\
& R: 5'-GACCACCTCAGAGATAGGTCC-3' \\
qPCR, DANCR & F: 5'-TTGTATGGGTGCATGTTCAGC-3' \\
& R: 5'-TAAGTCAATTGAAATACCAGC-3' \\
\hline
\end{tabular}

$F$, forward; $\mathrm{PPCR}$, real-time quantitative $P C R ; R$, reverse.

vector (Clontech, Mountain View, CA). Renilla luciferase (Rluc)-SOX2 $3^{\prime}$ untranslated region (UTR) and Rluc-ARF1 DANCR biding site (DBS) were constructed by using endonuclease to digest the pLuc luciferase vector (Ambion Inc., Austin, TX) carrying the Rluc reporter gene and ligating it to the corresponding fragment, which encodes ARF1 DBS mRNA and human SOX2 3' UTR. By using a cDNA library from NP69 cells as a template, the SOX2 $3^{\prime}$ UTR and ARF1 DBS mRNA fragments were amplified by PCR method.

shRNA Knockdown, Single-Guide RNA Knockout, and Transfection Assays

shRNA knockdown, single-guide RNA knockout, and transfection assays were performed, as previously described. ${ }^{17}$ The single-guide RNA sequence of RBM3 was designed by utilizing the MIT online tool (http:// crispr.mit.edu, last accessed May 10, 2019). shRNA sequences were obtained from Shanghai Bioegene Co., Ltd. (Shanghai, China). shRNA sequence and packaging plasmids were transfected into HEK293 cells and used to generate viral particles. At 48 and 72 hours after transfection, the supernatants were collected and filtered through a $0.22-\mu \mathrm{m}$ membrane (Millipore, Billerica, MA). Viruses were then concentrated. CNE-2 or HNE-1 cells were then infected with these shRNA or small hairpin green fluorescence protein (shGFP) control viruses in the presence of $8 \mu \mathrm{g} / \mathrm{mL}$ polybrene. Infected cells were enriched by selection with $5 \mu \mathrm{g} / \mathrm{mL}$ puromycin 48 hours after infection. Multiple monoclonal cultures were screened for shRNA integration and activity by Western blot and real-time PCR.

\section{In Situ Hybridization}

By using the RNAscope 2.5 HD Detection ReagentBROWN kit (ACDBio, Newark, CA), in situ analysis of $D A N C R$ interactions was performed on paraffin-embedded sections. All analyses were performed on the basis of the manufacturer's instructions by utilizing a $D A N C R$-specific probe purchased from ACDBio. DANCR expression was 
quantified using a visual grading system as follows: the percentage of positive tumor cells on a scale of 0 to $4(0$, none; $1,1 \%$ to $25 \% ; 2,26 \%$ to $50 \% ; 3,51 \%$ to $75 \%$; and 4 , $>75 \%$ ); and the intensity of staining (graded on a scale of 0 to 3 : 0 , no staining; 1 , weak staining; 2 , moderate staining; and 3 , strong staining). The product of the extent and intensity grades was used to define the DANCR expression.

\section{Cell Proliferation and Viability Assays}

Direct cell counting: HNE-1, CNE-1, and NP69 cells $\left(1 \times 10^{3}\right)$ were plated onto 96 -well plates with medium containing $10 \%$ fetal bovine serum, which were defined as the time of 0 hour. After 72 hours, cell numbers were counted and relative cell proliferation was calculated (72-hour number/0-hour number).

Cell counting kit-8 (CCK8) cell assay: HNE-1, CNE-1, and NP69 cells $\left(1 \times 10^{3}\right)$ were plated onto 96-well plates with medium containing $10 \%$ fetal bovine serum, and incubated with the CCK8 reagent. Cell viability was analyzed at the time of $0,24,48$, and 72 hours. The absorbance (OD) was measured at a wavelength of $490 \mathrm{~nm}$ using a Microplate Autoreader (Bio-Tek Instruments, Winooski, VT).

\section{RNA Pull-Down and RNA Immunoprecipitation Assays}

The RNA pull-down assays and RNA immunoprecipitation assays were conducted as previously reported. ${ }^{15}$ The relevant antibodies were listed as follows: SOX2 (catalog number 2748; 1:100; Cell Signaling Technology, Boston, MA) and RBM3 (catalog number ab211356; 1:100; Abcam, London, UK).

\section{RNA Transcriptome Sequencing}

In brief, the mRNA was utilized in construction of cDNA library and subsequent ion.

\section{Proton Sequencing}

On the basis of the manufacturer's instructions, the Ion Total RNA-Seq Kit version 2 (Lifetech, Carlsbad, CA; catalog number 4479789) was used to prepare the cDNA library. The Agilent Bioanalyzer 2200 (Agilent Technologies, Santa Clara, CA) was used to qualitatively assess and quantitate the cDNA libraries. After that, on the basis of the commercially available protocols, the cDNA libraries were processed by means of proton sequencing. According to instruction of Ion PI Template OT2 200 Kit (Lifetech; catalog number 4482286), samples were processed and enriched on a One Touch 2 ES station to prepare the template-positive Ion PI Ion Sphere Particles. Subsequently, 1 P1v2 Proton Chip was used to load the mixed templatepositive Ion PI Ion Sphere Particles of two samples and then sequenced on Proton Sequencers on the basis of the instruction of the Ion PI Sequencing 200 Kit (Lifetech; catalog number 4482283). RNA-Seq reads were mapped to the human reference genome sequence (hg38) assembly by Hisat2 version 2.1.0. ${ }^{18}$ Stringtie version $1.3 .3^{19}$ was used to count the number of fragments of each known gene downloaded from Ensembl (Homo sapiens GRCh38). After mapping, the genes with $<10$ fragments in all of the samples were removed and differentially expressed genes were identified using $\mathrm{R} /$ Bioconductor package edge $\mathrm{R}$ version 3.0.8. ${ }^{20}$ Thresholds of $P<0.05$ and fold change $\geq 1.5$ were used to identify differentially expressed genes. The results for RNA-Seq analysis are listed in Supplemental Table S1. Accession numbers are from National Center for Biotechnology Information Nucleotide database (https://www.ncbi. nlm.nih.gov/nuccore).

\section{Western Blot Analysis}

Western blot analysis was conducted as previously reported. ${ }^{17}$ The specific antibodies were as follows: RBM3 (catalog number ab211356; 1:1000; Abcam), SOX2 (cata$\log$ number 2748; 1:1000; Cell Signaling Technology), and glyceraldehyde-3-phosphate dehydrogenase (catalog number ab9485; 1:1000; Abcam).

\section{Xenografts}

In brief, female BALB/c-nude mice, 15 to $18 \mathrm{~g}$ (SLAC, Shanghai, China), were housed standardly. HNE-1 cells were collected, washed, and reconstituted with serum-free medium. After mixing with Matrigel (Becton-Dickinson, Franklin Lakes, NJ) in a 1:1 ratio, HNE-1 cells $\left(2 \times 10^{6}\right)$ were subcutaneously implanted into the right flank of each BALB/c-nude mouse. All experimental procedures were conducted according to institutional animal regulations. Relevant approvals were obtained from the Animal Care and Use Committee of the Zhejiang Provincial People's Hospital (Hangzhou, China). The IVIS Lumina imaging station (Caliper Life Sciences, Hopkinton, MA) was used to perform bioluminescence imaging. The anesthetic was 5\% chloral hydrate, and the dose used was according to the weight of mice $(0.5 \mathrm{~mL} / \mathrm{kg})$. The animals used in this study were euthanized by $\mathrm{CO}_{2}$ inhalation in separate euthanasia cases using $\mathrm{CO}_{2}$ gas cylinders.

\section{Statistical Analysis}

Statistical analyses were conducted in GraphPad Prism software version 5.0 (GraphPad Software, San Diego, CA). The $t$-test or $U$-test was utilized to determine the significance of the data between experimental groups in vitro and in vivo. The Pearson correlation coefficient was utilized to assess the data significance of specimens. A two-sided $P<0.05$ was considered statistically significant unless otherwise stated. 


\section{Results}

\section{DANCR Is Up-Regulated in NPC and Related with Poor Prognosis}

The data indicated that the expression of DANCR was markedly higher in five NPC cell lines (C666-1, HNE-1, SUNE-1, CNE-1, and CNE-2) than in the nasopharyngeal epithelial cell line (NP69) (Figure 1A). The expression of $D A N C R$ was also investigated in 10 pairs of freshly frozen NPC tumor tissues and adjacent normal tissues. As a result, the expression of DANCR was dramatically higher in NPC tumor tissues in comparison with that in the adjacent normal tissues (Figure 1B). RNA in situ hybridization was used to assess DANCR expression levels in 100 pairs of paraffin-embedded NPC tumor tissues and adjacent normal tissues. The result showed that DANCR expression was dramatically up-regulated in the tumor tissues (Figure 1, C and D), thereby confirming the real-time quantitative PCR result.

To investigate the clinical significance of $D A N C R$ expression in NPC, the effects of DANCR expression on the survival rates of NPC patients were evaluated. The high-DANCR patients exhibited lower overall survival and relapse-free rates than the low-DANCR patients (Figure 1E). In addition, NPC cells showed DANCR expression in both the cellular nucleus and cytoplasm (Figure 1F), which revealed that DANCR may function in both the NPC cell nucleus and cytoplasm. These data show that DANCR is dramatically up-regulated in NPC and is related with bad clinical outcome.

\section{Effects of DANCR on the Proliferation Growth and Tumorigenicity of NPC Cells}

To study whether DANCR is important for NPC growth and tumorigenicity, shRNAs were used to deplete endogenous DANCR in HNE-1 and CNE-2 cell lines (Figure 2A). Subsequent assays revealed that DANCR knockdown significantly inhibited cellular proliferation (Figure $2 \mathrm{~B}$ and Supplemental Figure S1, A and B) as well as colony formation (Figure 2, C and D). Moreover, DANCR knockdown also reduced NPC cell tumorigenicity (Figure 2, I and J). Conversely, overexpression of DANCR (Figure 2E and
A

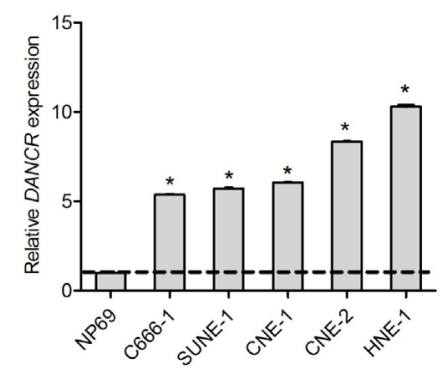

C

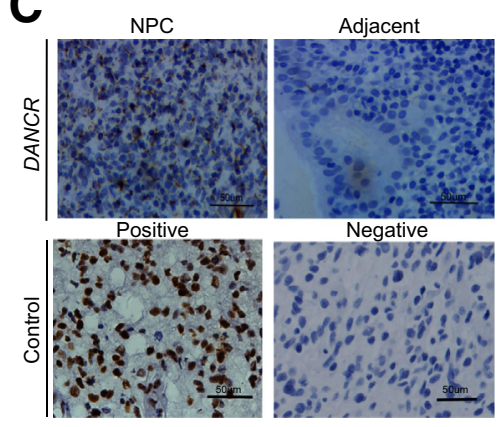

E

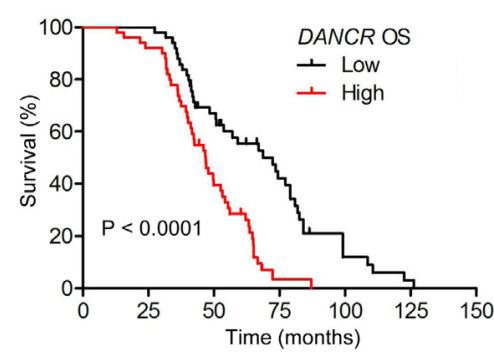

B

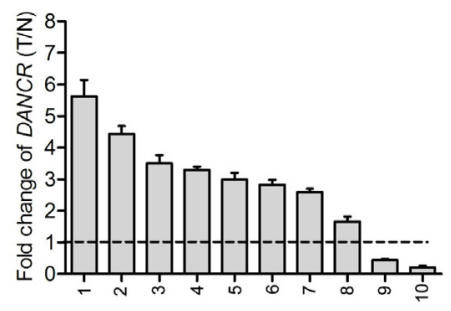

D
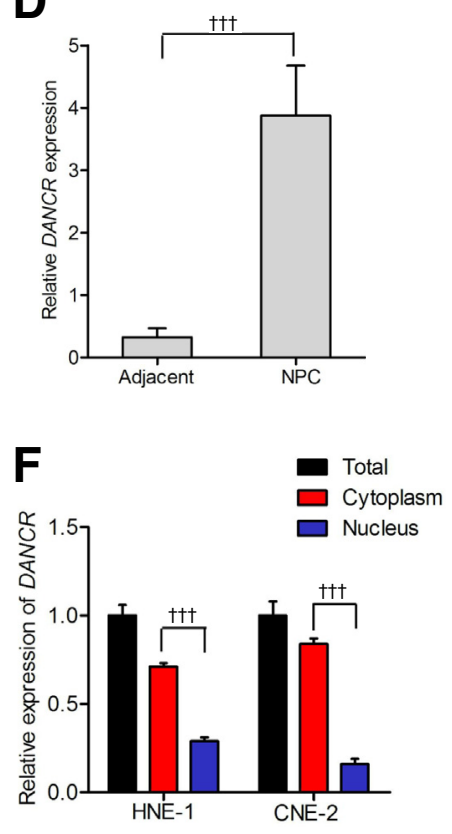

Figure 1 DANCR is up-regulated in NPC and associated with poor prognosis. A: DANCR is up-regulated in NPC cells compared with the nasopharyngeal epithelial cells. The dashed line represents DANCR expression in NP69 cell line. B: $D A N C R$ expression was detected by real-time quantitative PCR in 10 paired human NPC tissues and adjacent normal tissues. The dashed line represents the same expression of DANCR in NPC tissues and adjacent normal tissues. C: Representative images of DANCR expression in paraffin-embedded NPC tumors and paired adjacent nontumor specimens using RNAscope. Negative and positive control are shown. D: Quantification of DANCR expression in C. E: Kaplan-Meier analysis of overall survival (OS) based on DANCR expression in all the 100 patients. F: DANCR expression in the nucleus and cytoplasm of NPC cells. Data represent three independent experiments. Error bars represent the SD of each value $(\mathbf{A}$, B, D, and F). $n=100$ adjacent and NPC (D); $n=50$ low and high (E). ${ }^{*} P<0.05$ versus NP69; ${ }^{\dagger \dagger} P<0.001$. Scale bars $=50 \mu \mathrm{m}(\mathrm{C}) . \mathrm{T} / \mathrm{N}$, tumor/normal. 

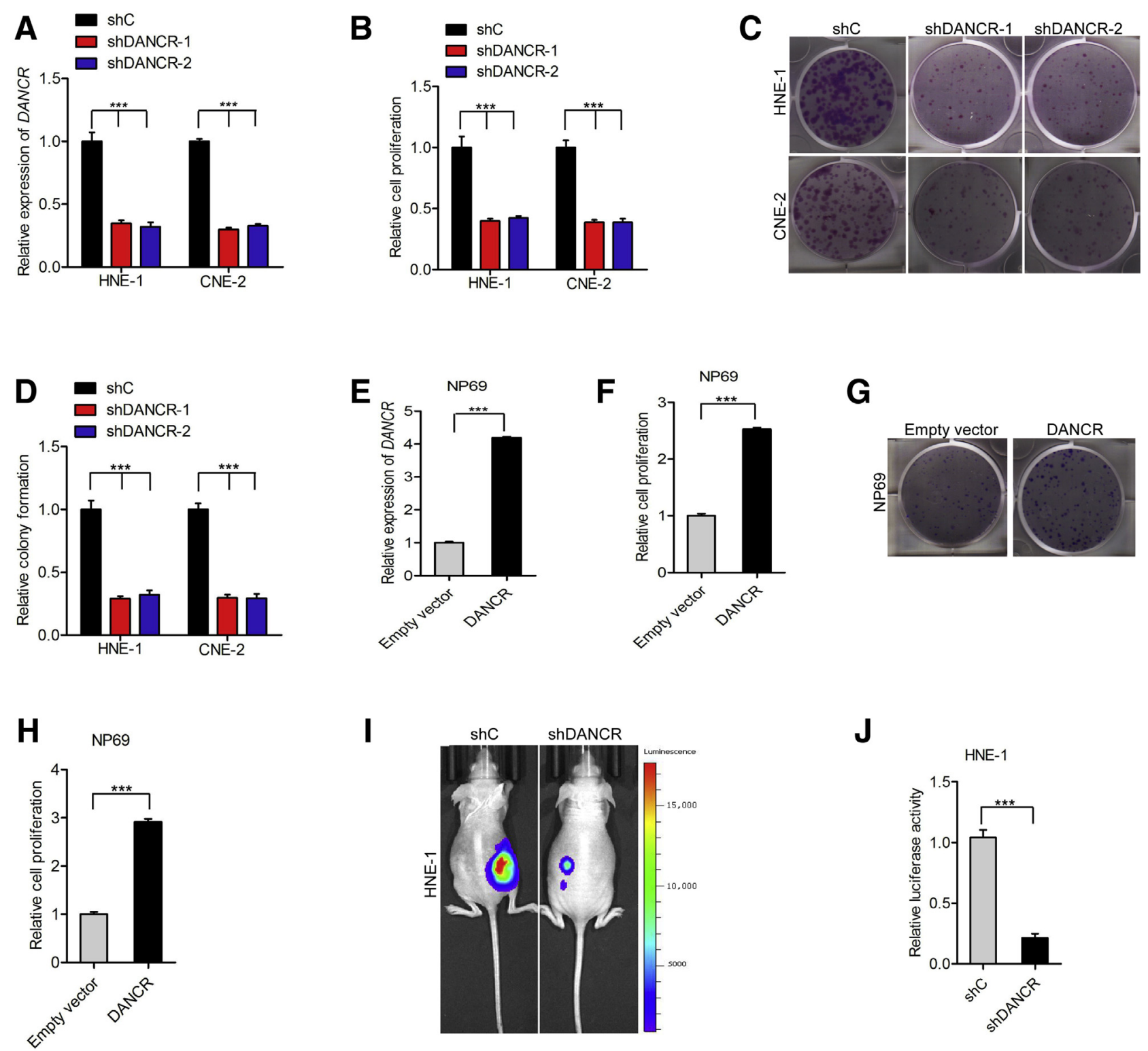

Figure 2 Effects of DANCR on the proliferation growth and tumorigenicity of NPC cells. A: Effects of DANCR knockdown in NPC cells. B and C: DANCR knockdown inhibits NPC cell proliferation (B) and colony formation (C). D: Quantification of colony formation in C. E: Effects of DANCR overexpression on NP69. F and G: DANCR overexpression promotes NP69 cell proliferation (F) and colony formation (G). H: Quantification of colony formation in G. I: Representative bioluminescence images following DANCR depletion-suppressing HNE-1 s.c. tumor growth. J: Quantification of the bioluminescence activity in I. Data represent three independent experiments. Error bars represent SD (A, B, D-F, H, and J). ${ }^{\star \star *} P<0.001$. shC, control without inhibition of DANCR expression; shDANCR, experimental group with inhibition of DANCR expression.

Supplemental Figure S1C) promoted cellular proliferation (Figure 2F) as well as colony formation (Figure 2, $\mathrm{G}$ and $\mathrm{H}$ ) in NP69. In conclusion, these data suggested that DANCR is required for NPC tumorigenicity and may be important in the transition of normal cells to tumor precursors.

\section{DANCR Regulates NPC Cell Proliferation by Stabilizing SOX2 mRNA}

To probe the DANCR-associated downstream genes, RNASeq analysis was performed to compare the mRNA expression pattern influenced by DANCR knockdown. Six- hundred and twenty-three differentially expressed genes were identified $(P<0.05$ and fold change $>1.5)$ (Figure $3 \mathrm{~A}$ and Supplemental Table S1) and six genes (SOX2, IGF1, LAMA4, NOS3, FGF19, and FGF13) were found to be down-regulated and five genes $(P 15, P 21$, $S P R Y 4, P L K 3$, and $K L F 2$ ) to be up-regulated by real-time quantitative PCR analysis on DANCR knockdown (Figure 3B). In addition, SOX2 was one of the top differentially expressed genes. SOX2 is required for NPC cell proliferation. ${ }^{16}$ We hypothesized that $D A N C R$ regulates NPC cell proliferation via SOX2. DANCR depletion inhibited SOX2 mRNA and protein expression (Figure 3, C 

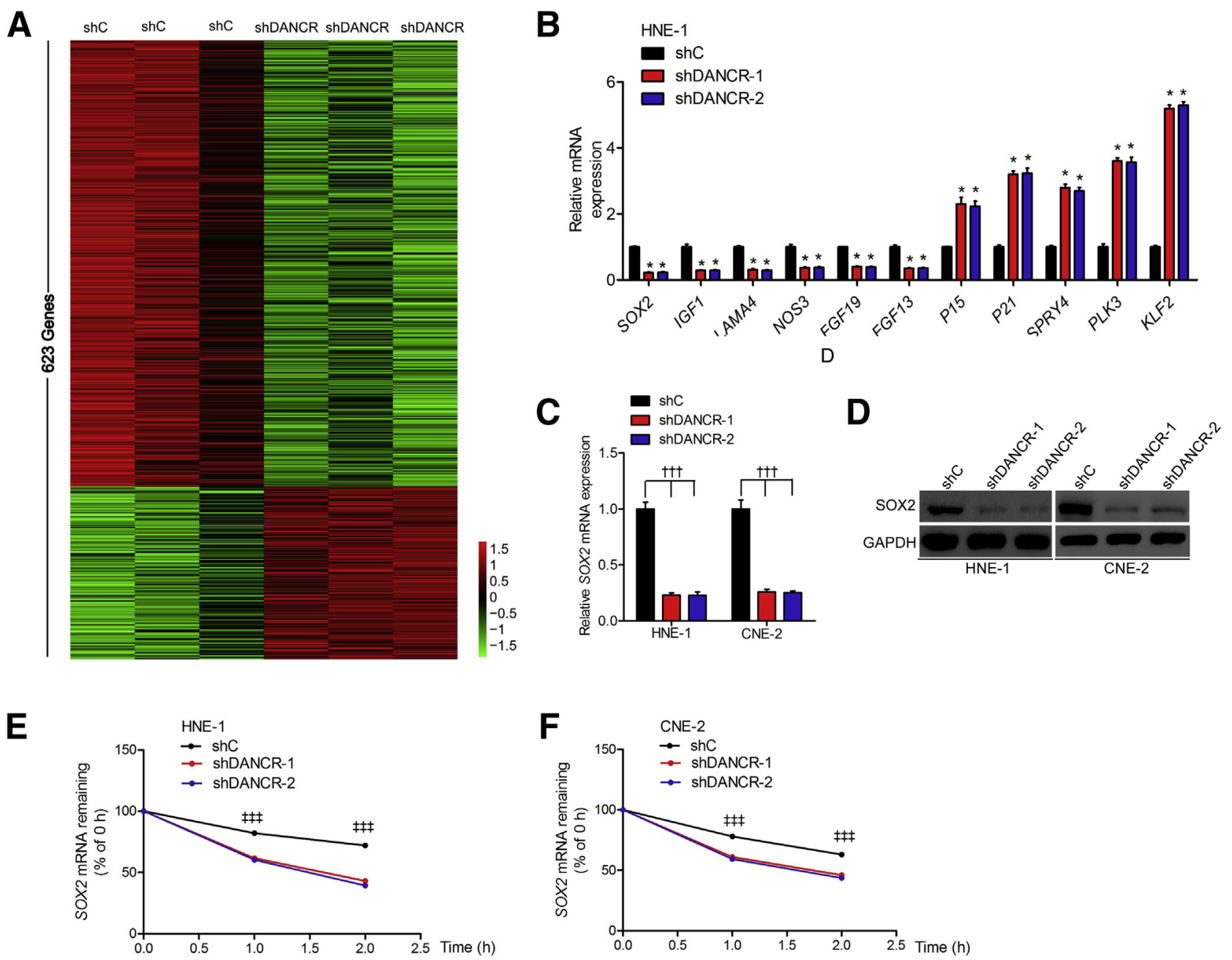

Figure 3 DANCR stabilizes SOX2 mRNA. A: Mean-centered, hierarchical clustering of 623 transcripts altered $(P<0.05$ and fold change $>1.5)$ in shCtreated cells and shDANCR-treated cells, with three repeats. B: Real-time quantitative PCR analyses in shC- versus shDANCR-treated NPC cells reveal altered mRNA levels of genes on DANCR depletion. $\mathbf{C}$ and $\mathbf{D}$ : DANCR depletion inhibits SOX2 mRNA (C) and protein (D) expression. E and F: DANCR controls SOX2 mRNA stability in NPC cells, which were treated with RNA synthesis inhibitor actinomycin D and analyzed at 0,1 , and 2 hours after exposure. Data represent three independent experiments. Error bars represent SD (B and $\mathbf{C})$. ${ }^{*} P<0.05$ versus shC; ${ }^{\dagger \dagger \dagger} P<0.001$; ${ }^{\ddagger \ddagger \ddagger} P<0.001$ shDANCR-1/shDANCR-2 versus shC at the same time point. GAPDH, glyceraldehyde-3-phosphate dehydrogenase; shC, control without inhibition of DANCR expression; shDANCR, experimental group with inhibition of DANCR expression.

and D), indicating that SOX2 may be involved in the $D A N C R$-mediated network. Further study suggested that SOX2 mRNA degraded more rapidly in the DANCR knockdown group compared with the control group (Figure 3, E and F), which was in agreement with a previous report. ${ }^{10}$

To further study the role of SOX2 in DANCR-regulated NPC proliferation, SOX2 was overexpressed in DANCRknockdown NPC cells (Figure 4, A and B). The overexpression restored $D A N C R$-inhibited cellular proliferation and colony formation (Figure 4, D-F). However, SOX2 overexpression could not restore DANCR-inhibited DANCR expression (Figure 4C). Conversely, SOX2 depletion also restored the cellular proliferation and colony formation promoted by DANCR overexpression (Figure 4, G-I). These data indicate that DANCR regulates NPC cell proliferation by stabilizing $S O X 2$ mRNA.

\section{DANCR Complexes with RBM3 and Influences SOX2 mRNA Stability}

RBM3 stabilizes $Y A P$ mRNA expression during cold stress. ${ }^{21}$ Hence, we studied whether SOX2 serves as a bona fide RBM3 target in NPC cells. RBM3 associated with SOX2 mRNA, using RNA immunoprecipitation assays (Figure 5A). The RNA pull-down assay further demonstrated that DANCR bonded with RBM3 protein (Figure 5B). Moreover, SOX2 mRNA expression significantly decreased in DANCRinhibited and RBM3-inhibited NPC cells (Figure 5C), suggesting that the DANCR-RBM3 complex may have affected $S O X 2$ mRNA expression. The following test plasmids were then transfected: the RBM3-FLAG expression vector, pLUC$S O X 2$ 3' UTR, pLUC-ARF1 RBM3-binding site, and phCMV-MUP reference plasmid that encodes urinary protein (MUP) mRNA. The latter two plasmids acted as positive and 

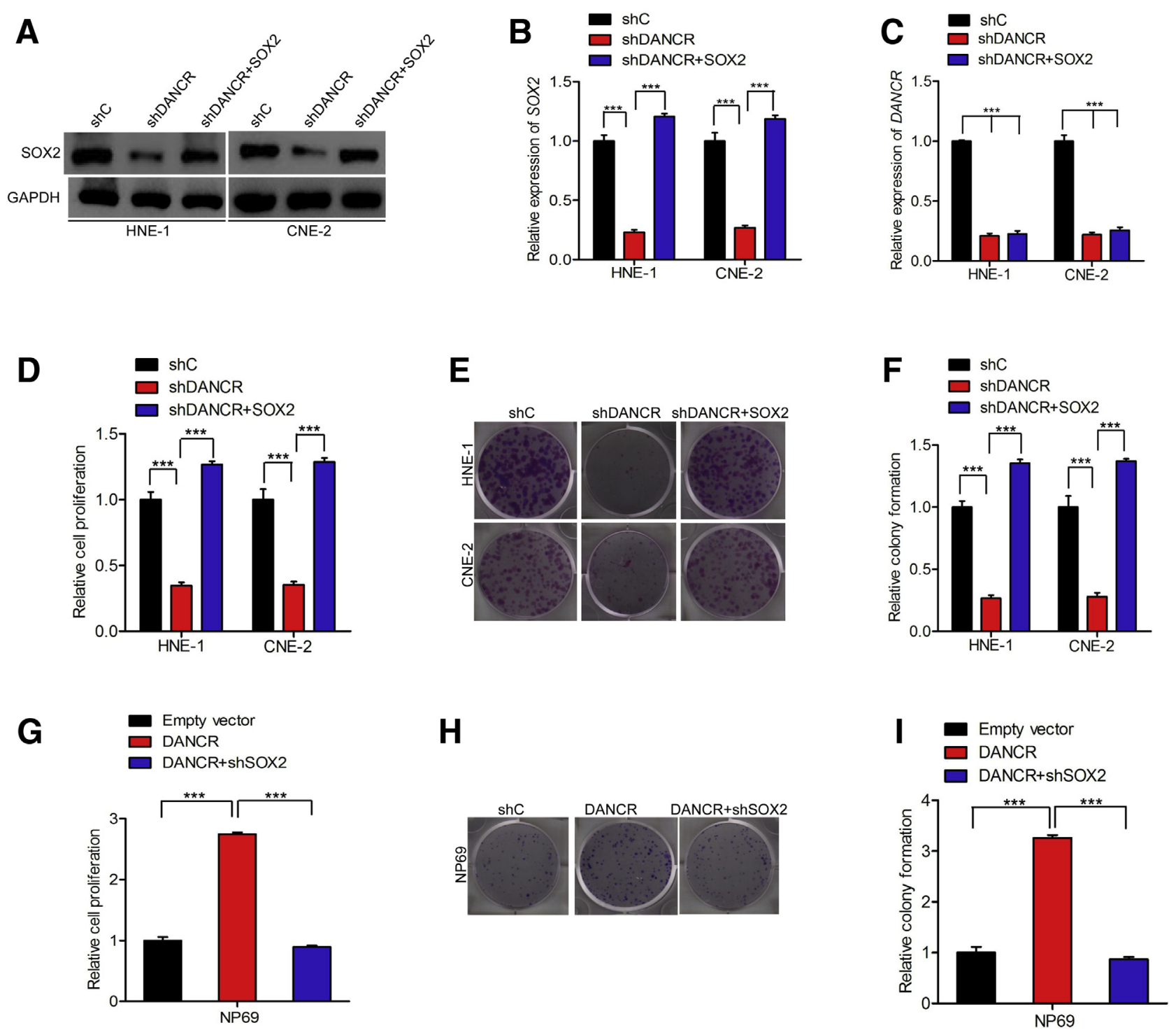

Figure 4 DANCR regulates NPC cell proliferation via SOX2. A and B: Effects of SOX2 overexpression in DANCR depletion-inhibited NPC cells. C: SOX2 overexpression had no effects on DANCR depletion-inhibited DANCR. D and E: SOX2 overexpression rescues DANCR depletion-inhibited NPC cell proliferation (D) and colony formation (E). F: Quantification of colony formation in E. $\mathbf{G}$ and $\mathbf{H}$ : SOX2 knockdown in DANCR overexpression background promotes NP69 cell proliferation $(\mathbf{G})$ and colony formation $(\mathbf{H})$. I: Quantification of colony formation in $\mathbf{H}$. Data represent three independent experiments. Error bars represent SD $\left(\mathbf{B}-\mathbf{D}, \mathbf{F}, \mathbf{G}\right.$, and I). ${ }^{* *} P<0.001$. GAPDH, glyceraldehyde-3-phosphate dehydrogenase; shC, control without inhibition of DANCR expression; shDANCR, experimental group with inhibition of DANCR expression.

negative controls, respectively, for RBM3-FLAG binding. Anti-FLAG antibodies were capable of immunopurifying Rluc-SOX2 3' UTR and Rluc-ARF1 RBM3-binding site, but not for MUP mRNA (Figure 5D). The data, taken together, suggest that $S O X 2$ is a bona fide $R B M 3$ target in NPC cells.

To reveal the role of DANCR in RBM3 association with SOX2 mRNA, a co-immunoprecipitation assay was performed. DANCR depletion inhibited RBM3 association with SOX2 mRNA (Figure 5E). Furthermore, DANCR interacted with SOX2 mRNA (Figure 5F) and RBM3 depletion inhibited the association of DANCR with SOX2 mRNA (Figure 5G), indicating that RBM3 was needed for the binding between $D A N C R$ and SOX2 mRNA. Moreover, DANCR or RBM3 depletion significantly decreased the half-life of SOX2 mRNA (Figure 5H). In conclusion, DANCR regulates SOX2 mRNA stability and expression using $R B M 3$.

\section{DANCR Promotes NPC Cell Proliferation via RBM3- Mediated SOX2}

$D A N C R, R B M 3$, and SOX $2 \mathrm{mRNAs}$ were demonstrated to be complexed. RBM3 was overexpressed in DANCR-depleted NPC cells. RBM3 overexpression restored DANCR 
A

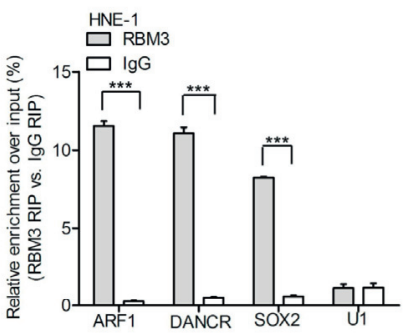

D

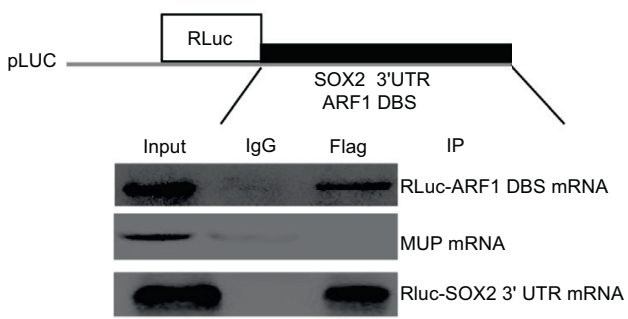

G

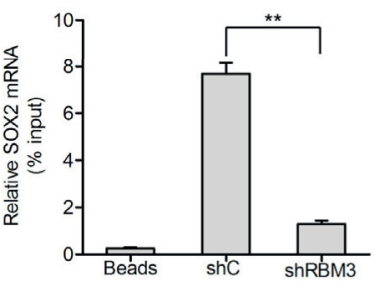

B

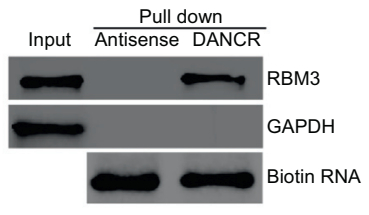

E

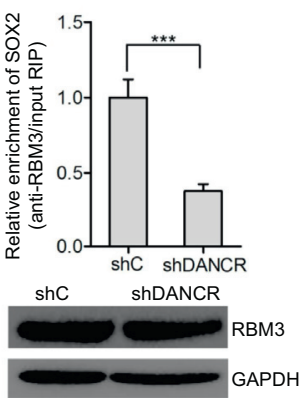

C

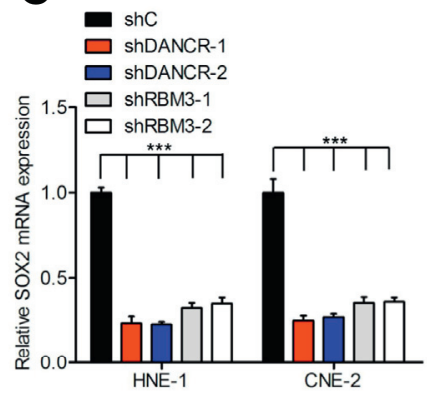

F

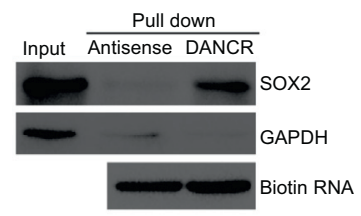

H

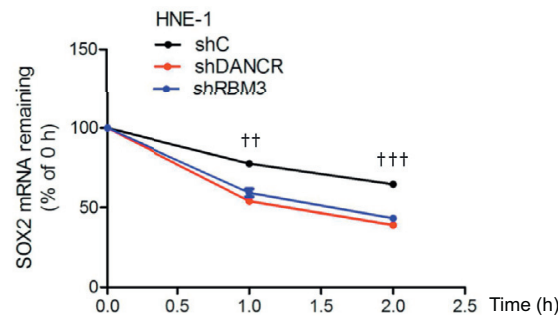

Figure 5 DANCR complexes with RBM3 and influences SOX2 mRNA stability. A: Interaction of DANCR or SOX2 mRNA with RBM3. B: RNA pull-down assay shows that DANCR binds to RBM3. C: DANCR or RBM3 depletion inhibits SOX2 mRNA expression in NPC cells. D: SOX2 $3^{\prime}$ untranslated regions (UTRs) and ARF1 DBS bind RBM3-FLAG, whereas MUP mRNA does not (below). Results are representative of three independently performed experiments. E: Inhibition of the interaction between SOX2 mRNA and RBM3 on DANCR suppression. F: RNA pull-down assay shows that DANCR binds to SOX2 mRNA. G: RBM3 depletion reduces the association between DANCR and SOX2 mRNA. H: DANCR or RBM3 control SOX2 mRNA stability in NPC cells. Data are representative of three independent experiments. Error bars represent SD (A, C, E, and G). ${ }^{* *} P<0.01,{ }^{*} * P<0.001 ;{ }^{\dagger \dagger} P<0.01,{ }^{\dagger \dagger} P<0.001$ shDANCR-1/shDANCR-2 versus shC. GAPDH, glyceraldehyde-3-phosphate dehydrogenase; IP, immunoprecipitation; RIP, RNA immunoprecipitation; shC, control without inhibition of DANCR expression; shDANCR, experimental group with inhibition of DANCR expression.

depletion-inhibited SOX2 protein, mRNA expression, cellular proliferation, and colony formation (Figure 6, A-D). However, DANCR did not restore RBM3 knockoutsuppressed SOX2 protein, mRNA expression, cellular proliferation, and colony formation (Figure 6, E-H), suggesting that $S O X 2$ regulation by $D A N C R$ relied on RBM3.

To confirm the clinical significance of our results, the mRNA expression levels of DANCR and SOX2 were detected in 100 clinical NPC tissues. DANCR significantly correlated with $S O X 2$ and RBM3 in the $\chi^{2}$ test (Figure 7, A and B). Moreover, co-expression of $D A N C R / S O X 2$ at high expression levels positively correlated with inferior prognosis in NPC patients (Figure 7C). These data support the conclusion that $D A N C R$ expression significantly correlates with $S O X 2$ in NPC.

\section{Discussion}

Although great advancements, such as combined chemoradiotherapy, intensity-modulated radiotherapy, and local and regional controls, have been made in the treatment of NPC, patient prognosis is still dismal, and this can be attributed to limited knowledge of the exact molecular pathogenesis as well as paucity of targeted therapies. ${ }^{22}$ Hence, unveiling the precise molecular switch that controls the malignant transformation of NPC is of great importance to identify novel prognostic indicators. This study revealed an unreported molecular mechanism by which the IncRNA DANCR stabilized SOX2 mRNA by interacting with RBM3, thereby activating SOX2-induced NPC tumorigenesis (Figure 7D). 


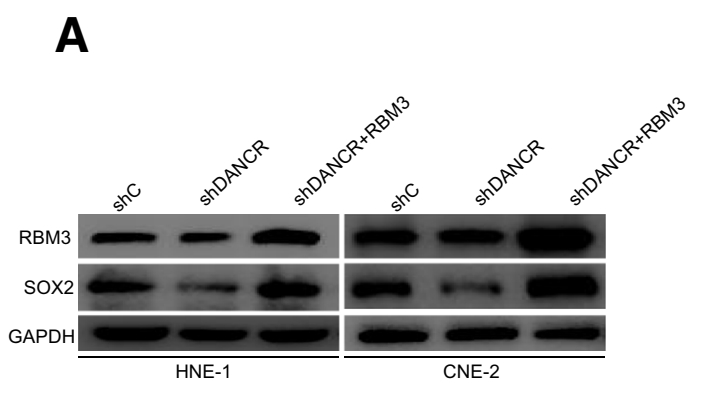

B

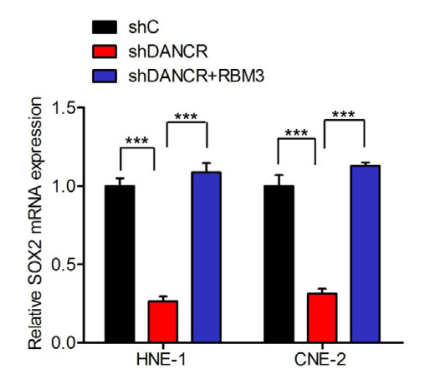

C

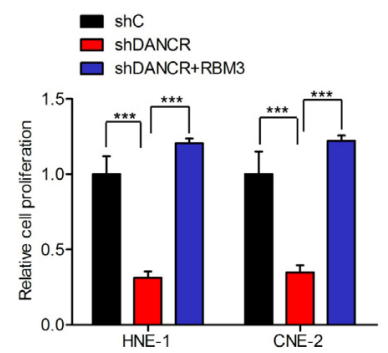

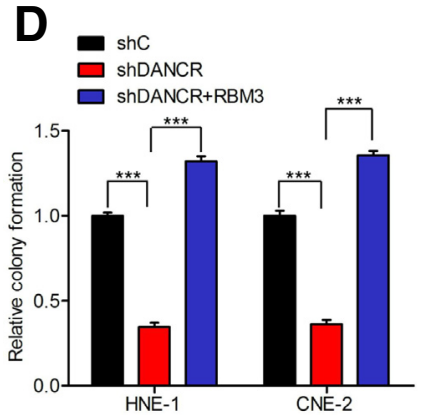

G

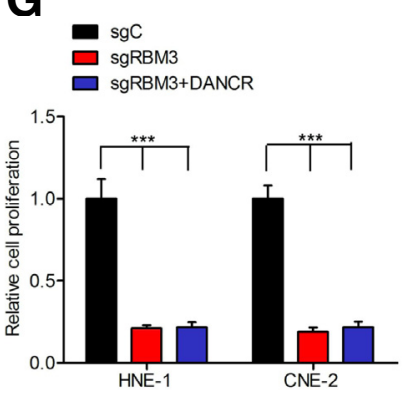

$\mathbf{E}$

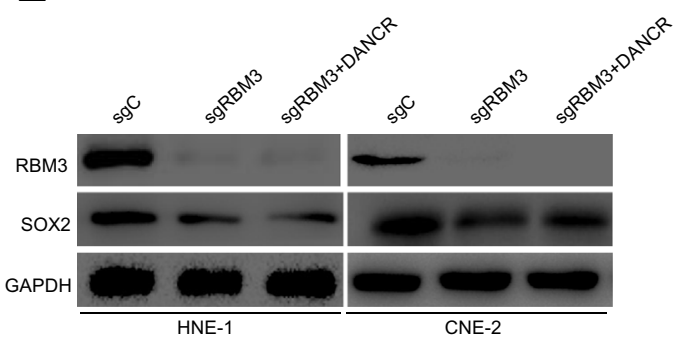

H

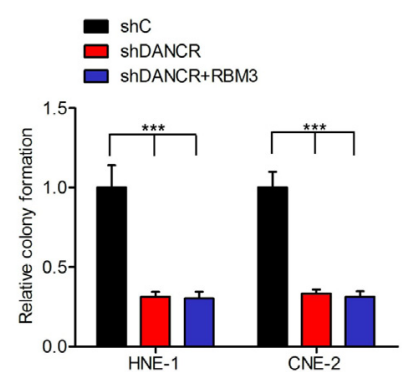

Figure 6 DANCR promotes NPC cell proliferation via RBM3-mediated SOX2. A and B: Effects of RBM3 overexpression on DANCR depletion-inhibited SOX2 protein (A) and mRNA (B) expression in NPC cells. C and D: RBM3 overexpression restored DANCR depletion-inhibited NPC cell proliferation (C) and colony formation (D). E and F: Effects of DANCR overexpression on RBM3 knockout-inhibited SOX2 protein (E) and mRNA (F) expression in NPC cells. G and H: DANCR overexpression restored RBM3 knockout-inhibited NPC cell proliferation $(\mathbf{G})$ and colony formation $(\mathbf{H})$. Data are representative of three independent experiments. Error bars represent SD $(\mathbf{B}-\mathbf{D}$ and $\mathbf{F}-\mathbf{H})$. ${ }^{* * *} P<0.001$. GAPDH, glyceraldehyde-3-phosphate dehydrogenase; sgC, control without knockout of RBM3 expression; sgRBM3, experimental group with knockout of RBM3 expression; shC, control without inhibition of DANCR expression; shDANCR, experimental group with inhibition of DANCR expression.

Aberrantly expressed IncRNAs were widely reported to be related with tumorigenesis and the development of various cancers, including NPC. ${ }^{17,23,24}$ For instance, Zheng et $\mathrm{al}^{25}$ demonstrated that the IncRNA FAM225A promotes NPC tumorigenesis and metastasis by acting as competing endogenous RNA to sponge miR-590-3p/miR-1275 and upregulate integrin beta chain beta 3 (ITGB3). In contrast, a recent study by Zhang et $\mathrm{al}^{26}$ revealed that the IncRNA NKILA represses the carcinogenesis and metastasis of NPC by NF- $\kappa \mathrm{B}$ pathway inhibition. Moreover, our previous findings also identified two lncRNAs (PVT1 and ANRIL) that play vital roles in nasopharyngeal carcinogenesis. ${ }^{5,27}$ However, the role of DANCR (which is a well-validated oncogenic lncRNA in multiple tumors) in NPC tumorigenesis and its underlying mechanisms remained largely unknown.

In this study, the DANCR expression in freshly frozen NPC tumor tissues was found to be dramatically higher than that in adjacent normal tissues, and was significantly associated with the patient prognosis, which was consistent with the results of earlier research. ${ }^{10}$ The oncogenic role of $D A N C R$ in NPC cell lines was also well established in this study. RNA-Seq analysis was utilized to explore the $D A N C R$-associated downstream genes. Various differentially expressed genes were identified and subsequently validated using real-time quantitative PCR analysis. SOX2 was found to be the most differentially expressed downstream gene. More important, SOX2 is a transcription factor 

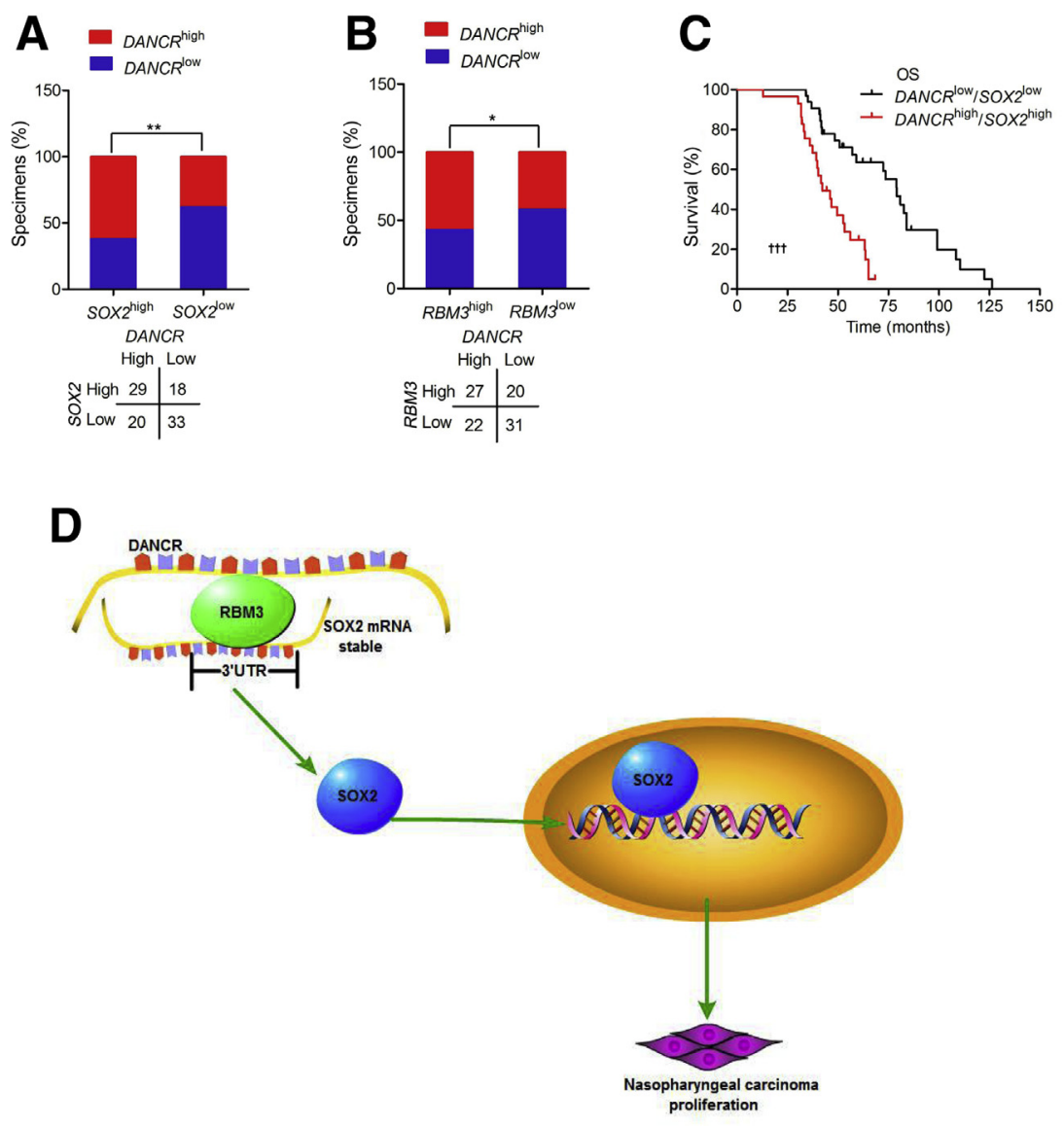

Figure 7 DANCR expression significantly corelates with SOX2 in NPC. A and B: Correlation of expression levels between DANCR and SOX2 (A) or RBM3 (B) mRNA. C: Prognosis comparison of NPC patients with $D A N C R / S O X 2$ ectopic differential expression using Kaplan-Meier survival analysis. D: Summary diagram describes that DANCR regulates NPC cell proliferation. $n=33 D A N C R^{\text {low }} / S_{S O X}{ }^{\text {low. }}$. $n=29 D A N C R^{\text {high }} /$ SOX $^{\text {high }} .{ }^{*} P<0.05$, ${ }^{* * P}<0.01 ;{ }^{\dagger \dagger \dagger} P<0.001$ overall OS of NPC patients with $D A N C R^{\text {low }} / \mathrm{SOX}^{\text {low }}$ versus overall OS of NPC patients with $D A N C R^{\text {high }} / S O 2^{\text {high }}$ (KaplanMeier survival analysis). OS, overall survival; shC, control without inhibition of DANCR expression; ShDANCR, experimental group with inhibition of DANCR expression; UTR, untranslated region. that has been widely reported to act as an oncogene in multiple types of malignant tumors. ${ }^{28-30}$ Interestingly, SOX2 mediates cell proliferation in NPC through the phosphatidylinositol 3-kinase/AKT signaling pathway. ${ }^{16}$ Hence, it seems reasonable to make an assumption that $D A N C R$ regulates NPC tumorigenesis using SOX2. The functional studies indicated that overexpression of $S O X 2$ restored the knockdown of DANCR-induced cellular proliferation. Conversely, SOX2 depletion was capable of restoring $D A N C R$ overexpression-induced cellular proliferation. Furthermore, SOX2 mRNA degraded rapidly when $D A N C R$ was knocked down. Therefore, our results suggest that $D A N C R$ regulates NPC cell proliferation by stabilizing $S O X 2$ mRNA.

$R B M 3$, a member of the highly conserved family of RNA binding proteins (RNABPs), encodes a protein that contains an RNA-recognition motif capable of binding to both DNA and RNA. ${ }^{31,32}$ By interacting with the UTRs of mRNAs, RBM3 protein is capable of either stabilizing or destabilizing target mRNA. ${ }^{33}$ Recently, emerging evidence has indicated that $R B M 3$ serves as an oncogene in various human tumors, including breast cancer, ${ }^{34}$ colorectal cancer, $^{35,36}$ and prostate cancer. ${ }^{37,38}$ In contrast, many studies have shown that $R B M 3$ is a tumor suppressor in other cancers. ${ }^{31,39}$ Hence, these data suggest that RBM3 exhibits tissue specificity. It has been reported that $R B M 3$ drives radioresistance in NPC by reducing apoptosis via the phosphatidylinositol 3-kinase/Akt/Bcl-2 signaling pathway. ${ }^{40}$ However, the exact genes that can be regulated by $R B M 3$ in NPC remain largely unknown. In this study, we performed an RNA immunoprecipitation assay followed by an RNA pull-down assay and found that RBM3 was associated with both $S O X 2$ and DANCR. Furthermore, the expression level of $S O X 2$ was significantly affected by the $D A N C R$-RBM3 complex. Our results also indicated that RBM3 could bind directly to the $3^{\prime}$ UTR of SOX2. Therefore, it was convincingly demonstrated that $D A N C R$ formed a complex with RBM3 to stabilize SOX2 mRNA, thus regulating cellular proliferation in NPC. Moreover, the clinical data also suggested that co-expression of DANCR/ $S O X 2$ at high levels was positively associated with poor clinical outcomes of patients with NPC.

In summary, we have identified that DANCR acts as an oncogenic lncRNA in NPC and have convincingly unveiled a novel mechanism by which $D A N C R$ stabilizes SOX2 mRNA by forming a complex with RBM3, which ultimately enhances tumorigenesis in NPC. These findings may aid the identification of novel biomarkers and provide a theoretical basis for precise medical treatment of NPC in the future. 


\section{Author Contributions}

Q.L., L.Z., Y.J., M.Z., J.W., and H.Z.: performed animal experiments and designed the study; Y.L. and G.Z.: performed Western blot analysis; L.Z., T.S., Y.Z., and X.L.: analyzed data and wrote the manuscript; J.T. and H.Z.: conceived and supervised the study, analyzed data, and wrote the manuscript. All authors interpreted data and reviewed and approved the manuscript.

\section{Supplemental Data}

Supplemental material for this article can be found at http://doi.org/10.1016/j.ajpath.2020.09.005.

\section{References}

1. Xiong W, Zeng ZY, Xia JH, Xia K, Shen SR, Li XL, Hu DX, Tan C, Xiang JJ, Zhou J, Deng H, Fan SQ, Li WF, Wang R, Zhou M, Zhu SG, Lü HB, Qian J, Zhang BC, Wang JR, Ma J, Xiao BY, Huang H, Zhang QH, Zhou YH, Luo XM, Zhou HD, Yang YX, Dai HP, Feng GY, Pan Q, Wu LQ, He L, Li GY: A susceptibility locus at chromosome 3p21 linked to familial nasopharyngeal carcinoma. Cancer Res 2004, 64:1972-1974

2. Zeng Z, Huang H, Zhang W, Xiang B, Zhou M, Zhou Y, Ma J, Yi M, Li X, Li X, Xiong W, Li G: Nasopharyngeal carcinoma: advances in genomics and molecular genetics. Sci China Life Sci 2011, 54: 966-975

3. He Y, Jing Y, Wei F, Tang Y, Yang L, Luo J, Yang P, Ni Q, Pang J, Liao Q, Xiong F, Guo C, Xiang B, Li X, Zhou M, Li Y, Xiong W, Zeng Z, Li G: Long non-coding RNA PVT1 predicts poor prognosis and induces radioresistance by regulating DNA repair and cell apoptosis in nasopharyngeal carcinoma. Cell Death Dis 2018, 9:235

4. Tang L, Wei F, Wu Y, He Y, Shi L, Xiong F, Gong Z, Guo C, Li X, Deng H, Cao K, Zhou M, Xiang B, Li X, Li Y, Li G, Xiong W, Zeng Z: Role of metabolism in cancer cell radioresistance and radiosensitization methods. J Exp Clin Cancer Res 2018, 37:87

5. Wang Y, Chen W, Lian J, Zhang H, Yu B, Zhang M, Wei F, Wu J, Jiang J, Jia Y, Mo F, Zhang S, Liang X, Mou X, Tang J: The lncRNA PVT1 regulates nasopharyngeal carcinoma cellular proliferation via activating the KAT2A acetyltransferase and stabilizing HIF-1 $\alpha$. Cell Death Differ 2020, 27:695-710

6. Chen G, Sun W, Hua X, Zeng W, Yang L: Long non-coding RNA FOXD2-AS1 aggravates nasopharyngeal carcinoma carcinogenesis by modulating miR-363-5p/S100A1 pathway. Gene 2018, 645: $76-84$

7. Zhang W, Du M, Wang T, Chen W, Wu J, Li Q, Tian X, Qian L, Wang Y, Peng F, Fei Q, Chen J, He X, Yin L: Long non-coding RNA LINC01133 mediates nasopharyngeal carcinoma tumorigenesis by binding to YBX1. Am J Cancer Res 2019, 9:779-790

8. Zhang KJ, Tan XL, Guo L: The long non-coding RNA DANCR regulates the inflammatory phenotype of breast cancer cells and promotes breast cancer progression via EZH2-dependent suppression of SOCS3 transcription. Mol Oncol 2020, 14:309-328

9. Yuan SX, Wang J, Yang F, Tao QF, Zhang J, Wang LL, Yang Y, Liu H, Wang ZG, Xu QG, Fan J, Liu L, Sun SH, Zhou WP: Long noncoding RNA DANCR increases stemness features of hepatocellular carcinoma by derepression of CTNNB1. Hepatology 2016, 63: 499-511

10. Wen X, Liu X, Mao YP, Yang XJ, Wang YQ, Zhang PP, Lei Y, Hong XH, He QM, Ma J, Liu N, Li YQ: Long non-coding RNA DANCR stabilizes HIF- $1 \alpha$ and promotes metastasis by interacting with NF90/NF45 complex in nasopharyngeal carcinoma. Theranostics 2018, 8:5676-5689

11. Kretz M, Webster DE, Flockhart RJ, Lee CS, Zehnder A, LopezPajares V, Qu K, Zheng GX, Chow J, Kim GE, Rinn JL, Chang HY, Siprashvili Z, Khavari PA: Suppression of progenitor differentiation requires the long noncoding RNA ANCR. Genes Dev 2012, 26:338-343

12. Zhen Q, Gao LN, Wang RF, Chu WW, Zhang YX, Zhao XJ, Lv BL, Liu JB: LncRNA DANCR promotes lung cancer by sequestering miR-216a. Cancer Control 2018, 25. 1073274818769849

13. Liang H, Zhang C, Guan H, Liu J, Cui Y: LncRNA DANCR promotes cervical cancer progression by upregulating ROCK1 via sponging miR-335-5p. J Cell Physiol 2019, 234:7266-7278

14. Lu Y, Hu Z, Mangala LS, Stine ZE, Hu X, Jiang D, Xiang Y, Zhang Y, Pradeep S, Rodriguez-Aguayo C, Lopez-Berestein G, DeMarzo AM, Sood AK, Zhang L, Dang CV: MYC targeted long noncoding RNA DANCR promotes cancer in part by reducing p21 levels. Cancer Res 2018, 78:64-74

15. Tang J, Zhong G, Zhang H, Yu B, Wei F, Luo L, Kang Y, Wu J, Jiang J, Li Y, Wu S, Jia Y, Liang X, Bi A: LncRNA DANCR upregulates PI3K/AKT signaling through activating serine phosphorylation of RXRA. Cell Death Dis 2018, 9:1167

16. Tang J, Zhong G, Wu J, Chen H, Jia Y: SOX2 recruits KLF4 to regulate nasopharyngeal carcinoma proliferation via PI3K/AKT signaling. Oncogenesis 2018, 7:61

17. Zheng YJ, Zhao JY, Liang TS, Wang P, Wang J, Yang DK, Liu ZS Long noncoding RNA SMAD5-AS1 acts as a microRNA-106a-5p sponge to promote epithelial mesenchymal transition in nasopharyngeal carcinoma. FASEB J 2019, 33:12915-12928

18. Kim D, Paggi JM, Park C, Bennett C, Salzberg SL: Graph-based genome alignment and genotyping with HISAT2 and HISAT-genotype. Nat Biotechnol 2019, 37:907-915

19. Kovaka S, Zimin AV, Pertea GM, Razaghi R, Salzberg SL, Pertea M: Transcriptome assembly from long-read RNA-seq alignments with StringTie2. Genome Biology 2019, 20:278

20. Robinson MD, McCarthy DJ, Smyth GK: edgeR: a Bioconductor package for differential expression analysis of digital gene expression data. Bioinformatics 2010, 26:139-140

21. Xia W, Su L, Jiao J: Cold-induced protein RBM3 orchestrates neurogenesis via modulating Yap mRNA stability in cold stress. J Cell Biol 2018, 217:3464-3479

22. Lee AW, Ma BB, Ng WT, Chan AT: Management of nasopharyngeal carcinoma: current practice and future perspective. J Clin Oncol 2015 , 33:3356-3364

23. Hu W, Xu W, Shi Y, Dai W: IncRNA HOTAIR upregulates COX-2 expression to promote invasion and migration of nasopharyngeal carcinoma by interacting with miR-101. Biochem Biophys Res Commun 2018, 505:1090-1096

24. Peng J, Liu F, Zheng H, Wu Q, Liu S: Long noncoding RNA ZFAS1 promotes tumorigenesis and metastasis in nasopharyngeal carcinoma by sponging miR-892b to up-regulate LPAR1 expression. J Cell Mol Med 2020, 24:1437-1450

25. Zheng ZQ, Li ZX, Zhou GQ, Lin L, Zhang LL, Lv JW, Huang XD, Liu RQ, Chen F, He XJ, Kou J, Zhang J, Wen X, Li YQ, Ma J, Liu N, Sun Y: Long noncoding RNA FAM225A promotes nasopharyngeal carcinoma tumorigenesis and metastasis by acting as ceRNA to sponge miR-590-3p/miR-1275 and upregulate ITGB3. Cancer Res 2019, 79:4612-4626

26. Zhang W, Guo Q, Liu G, Zheng F, Chen J, Huang D, Ding L, Yang X, Song E, Xiang Y, Yao H: NKILA represses nasopharyngeal carcinoma carcinogenesis and metastasis by NF-kappaB pathway inhibition. PLoS Genet 2019, 15:e1008325

27. Wu J-H, Tang J-M, Li X-W: Upregulation of SOX2-activated lncRNA ANRIL promotes nasopharyngeal carcinoma cell growth. Sci Rep 2018, 8:3333

28. Lu Y, Futtner C, Rock JR, Xu X, Whitworth W, Hogan BL, Onaitis MW: Evidence that SOX2 overexpression is oncogenic in the lung. PLoS One 2010, 5:e11022 
29. Gen Y, Yasui K, Zen Y, Zen K, Dohi O, Endo M, Tsuji K, Wakabayashi N, Itoh Y, Naito Y, Taniwaki M, Nakanuma Y, Okanoue T, Yoshikawa T: SOX2 identified as a target gene for the amplification at $3 \mathrm{q} 26$ that is frequently detected in esophageal squamous cell carcinoma. Cancer Genet Cytogenet 2010, 202: 82-93

30. Maurizi G, Verma N, Gadi A, Mansukhani A, Basilico C: Sox2 is required for tumor development and cancer cell proliferation in osteosarcoma. Oncogene 2018, 37:4626-4632

31. Ehlén A, Brennan DJ, Nodin B, O'Connor DP, Eberhard J, AlvaradoKristensson M, Jeffrey IB, Manjer J, Brändstedt J, Uhlén M, Pontén F, Jirström K: Expression of the RNA-binding protein RBM3 is associated with a favourable prognosis and cisplatin sensitivity in epithelial ovarian cancer. J Transl Med 2010, 8:78

32. Wright CF, Oswald BW, Dellis S: Vaccinia virus late transcription is activated in vitro by cellular heterogeneous nuclear ribonucleoproteins. J Biol Chem 2001, 276:40680-40686

33. Burd CG, Dreyfuss G: Conserved structures and diversity of functions of RNA-binding proteins. Science 1994, 265:615-621

34. Jögi A, Brennan DJ, Rydén L, Magnusson K, Fernö M, Stål O, Borgquist S, Uhlen M, Landberg G, Påhlman S, Pontén F, Jirström K: Nuclear expression of the RNA-binding protein RBM3 is associated with an improved clinical outcome in breast cancer. Mod Pathol 2009, 22:1564-1574

35. Melling N, Simon $R$, Mirlacher $M$, Izbicki JR, Stahl $P$, Terracciano LM, Bokemeyer C, Sauter G, Marx AH: Loss of RNAbinding motif protein 3 expression is associated with right-sided localization and poor prognosis in colorectal cancer. Histopathology 2016, 68:191-198

36. Hjelm B, Brennan DJ, Zendehrokh N, Eberhard J, Nodin B, Gaber A, Pontén F, Johannesson H, Smaragdi K, Frantz C, Hober S, Johnson LB, Påhlman S, Jirström K, Uhlen M: High nuclear RBM3 expression is associated with an improved prognosis in colorectal cancer. Proteomics Clin Appl 2011, 5: 624-635

37. Jonsson L, Gaber A, Ulmert D, Uhlén M, Bjartell A, Jirström K: High RBM3 expression in prostate cancer independently predicts a reduced risk of biochemical recurrence and disease progression. Diagn Pathol 2011, 6:91

38. Grupp K, Wilking J, Prien K, Hube-Magg C, Sirma H, Simon R, Steurer S, Budäus L, Haese A, Izbicki J, Sauter G, Minner S, Schlomm T, Tsourlakis MC: High RNA-binding motif protein 3 expression is an independent prognostic marker in operated prostate cancer and tightly linked to ERG activation and PTEN deletions. Eur J Cancer 2014, 50:852-861

39. Boman K, Segersten U, Ahlgren G, Eberhard J, Uhlén M, Jirström K, Malmström PU: Decreased expression of RNA-binding motif protein 3 correlates with tumour progression and poor prognosis in urothelial bladder cancer. BMC Urol 2013, 13:17

40. Ma R, Zhao LN, Yang H, Wang YF, Hu J, Zang J, Mao JG, Xiao JJ, Shi M: RNA binding motif protein 3 (RBM3) drives radioresistance in nasopharyngeal carcinoma by reducing apoptosis via the PI3K/AKT/Bcl-2 signaling pathway. Am J Transl Res 2018, 10: $4130-4140$ 